\title{
ON WARING'S PROBLEM: THREE CUBES AND A MINICUBE
}

\author{
JÖRG BRÜDERN AND TREVOR D. WOOLEY
}

\begin{abstract}
We establish that almost all natural numbers $n$ are the sum of four cubes of positive integers, one of which is no larger than $n^{5 / 36}$. The proof makes use of an estimate for a certain eighth moment of cubic exponential sums, restricted to minor arcs only, of independent interest.
\end{abstract}

\section{§1. Introduction}

It was shown by Davenport [6] in 1939 that almost all natural numbers are the sum of four positive integral cubes, and it is now known that when $N$ is large, the number of positive integers not exceeding $N$ that fail to be thus represented is slightly smaller than $N^{37 / 42}$ (see [11], [3], [4], [16], and [17] for the most recent developments). Since integers congruent to 4 modulo 9 are never the sum of three cubes, this conclusion cannot be refined to one involving fewer summands. A formal application of the circle method predicts an asymptotic formula for the number of representations as the sum of four positive cubes, and this would imply that all large integers are thus represented. Indeed, the same heuristic argument suggests that the fourth cube is almost redundant, in that it may be replaced by a cube from a sparse sequence without impairing such conclusions. The purpose of this article is to investigate representations by sums of four positive cubes, one of which is small.

When $n$ is a natural number and $0<\theta \leq 1 / 3$, we denote by $r_{\theta}(n)$ the number of representations of $n$ in the form

$$
n=x_{1}^{3}+x_{2}^{3}+x_{3}^{3}+x_{4}^{3}
$$

Received August 31, 2009. Revised February 9, 2010. Accepted February 14, 2010. 2000 Mathematics Subject Classification. Primary 11P05, 11P55.

Wooley's work supported by a Royal Society Wolfson Research Merit Award.

(C) 2011 by The Editorial Board of the Nagoya Mathematical Journal 
with $x_{1}, x_{2}, x_{3}, x_{4}$ natural numbers satisfying $x_{4} \leq n^{\theta}$. As intimated above, one anticipates that $r_{\theta}(n) \sim \Gamma(4 / 3)^{3} \mathfrak{S}(n) n^{\theta}$, where $\mathfrak{S}(n)$ is the familiar singular series associated with sums of four cubes. We recall in this context that $\mathfrak{S}(n) \gg 1$ (see, e.g., [14, Section 4.6, Exercise 3]), and hence it is expected that $r_{\theta}(n) \geq 1$ when $n$ is large. We are able to confirm this expectation for values of $\theta$ rather smaller than $1 / 7$, at least, for almost all $n$.

THEOREM 1.1. When $\theta \geq 5 / 36$, one has $r_{\theta}(n) \geq 1$ for almost all $n$.

The methods of this article are capable of showing that the conclusion of this theorem remains valid when $\theta$ is slightly smaller than 0.13884 , whereas $5 / 36>0.13888$. For comparison, it is apparent that whenever $n$ is represented in the shape $(1.1)$, then $x_{i} \leq n^{1 / 3}(1 \leq i \leq 4)$, and so the conclusion is trivial for $\theta \geq 1 / 3$. One may interpret Theorem 1.1 as asserting that almost all large integers possess a (formal) representation as the sum of at most 3.417 positive cubes. Meanwhile, [5, Theorem 1] shows that almost all large integers not congruent to 5 modulo 9 are the sum of three positive integral cubes and a sixth power, a conclusion tantamount to one involving 3.5 cubes.

In Section 6, we show that the anticipated asymptotic formula holds almost always for sums of three cubes and a minicube.

TheOREM 1.2. Suppose that $1 / 4<\theta \leq 1 / 3$. Then, for almost all $n$, one has

$$
r_{\theta}(n)=\Gamma\left(\frac{4}{3}\right)^{3} \mathfrak{S}(n) n^{\theta}+O\left(n^{\theta}(\log n)^{-1}\right) .
$$

A conclusion equivalent to this theorem in the unrestricted situation with $\theta=1 / 3$ can be extracted from Vaughan [11, Theorem 3] by incorporating refinements due to Boklan [1]. We remark that it is unnecessary to restrict the minicube implicit in the representation of $n$ in Theorem 1.2 to be bounded by a pure power $n^{\theta}$, and that this hypothesis may be removed with some technical elaboration of our basic argument.

We prove Theorems 1.1 and 1.2 using the Hardy-Littlewood method, beginning in Section 2 with some auxiliary mean value estimates. In Section 3, we establish an upper bound for a certain eighth moment of cubic exponential sums restricted to minor arcs, an estimate of independent interest (see Theorem 3.1 for details). We lay the foundations for an application of the circle method in Section 4, deriving a lower bound for the contribution of the major arcs. Then, in Section 5, we apply Bessel's inequality to relate the exceptional set to a minor arc estimate. Following two pruning 
processes, the proof of Theorem 1.1 is complete. Although complicated by the limited availability of full-length generating functions to be applied in the analysis of the major arcs, the proof of Theorem 1.2 in Section 6 is essentially routine. Finally, in Section 7, we briefly discuss the representation of large natural numbers as the sum of seven positive integral cubes, one of which is restricted to be a minicube.

Throughout, we reserve the letter $\varepsilon$ to denote a sufficiently small positive number, and we use $P$ to denote a positive number sufficiently large in terms of $\varepsilon$. The implicit constants in Vinogradov's well-known notation $\ll$ and $\gg$ will depend at most on $\varepsilon$, unless otherwise indicated. Whenever $\varepsilon$ appears in a statement, either implicitly or explicitly, we assert that the statement holds for each $\varepsilon>0$. Note that the "value" of $\varepsilon$ may consequently change from statement to statement. Finally, throughout this article, a variable denoted by the letter $p$ should be interpreted as denoting a prime number congruent to 2 modulo 3 .

\section{$\S 2$. Catalytic lemmata}

As is to be expected when considering problems in which one or more variables are shortened, mean values involving diminishing ranges play a prominent role in the arguments that follow. We collect together these basic estimates in this section, and we begin now by introducing the cast of exponential sums. We take $P$ to be our basic parameter, a large real number, and then take $Q$ to be an auxiliary parameter with $1 \leq Q \leq P$, to be chosen in due course. Next, we define

$$
f(\alpha)=\sum_{P<x \leq 2 P} e\left(\alpha x^{3}\right) \quad \text { and } \quad g(\alpha)=\sum_{Q<y \leq 2 Q} e\left(\alpha y^{3}\right),
$$

where, here and throughout, we write $e(z)$ for $e^{2 \pi i z}$. Also, with $\eta$ fixed to be a sufficiently small positive number, we define the set of $X^{\eta}$-smooth numbers not exceeding $X$ by

$$
\mathcal{A}(X)=\left\{n \in[1, X] \cap \mathbb{Z}: \varpi \text { prime and } \varpi \mid n \Rightarrow \varpi \leq X^{\eta}\right\}
$$

We then take $R$ to be a positive number with $R \leq Q$, and we put

$$
h(\alpha)=\sum_{z \in \mathcal{A}(R)} e\left(\alpha z^{3}\right) .
$$


We record for future reference the estimate

$$
\int_{0}^{1}|h(\alpha)|^{6} d \alpha \ll R^{13 / 4-\tau}
$$

which holds for any positive number $\tau$ with $\tau^{-1}>852+16 \sqrt{2833}=$ 1703.6..., as a consequence of [17, Theorem 1.2].

Our purpose in this section is to record estimates for the three mean values

$$
\begin{aligned}
& T_{1}=\int_{0}^{1}\left|f(\alpha)^{2} g(\alpha)^{2} h(\alpha)^{6}\right| d \alpha, \\
& T_{2}=\int_{0}^{1}\left|f(\alpha)^{2} g(\alpha)^{4}\right| d \alpha, \\
& T_{3}=\int_{0}^{1}|f(\alpha) g(\alpha) h(\alpha)|^{2} d \alpha,
\end{aligned}
$$

of use in our subsequent deliberations. We begin with an analysis of $T_{1}$ via an auxiliary estimate.

LEMmA 2.1. Whenever $R \leq Q^{2 / 3}$, one has

$$
\int_{0}^{1}\left|g(\alpha)^{2} h(\alpha)^{6}\right| d \alpha \ll Q R^{13 / 4-\tau} .
$$

Proof. On considering the underlying diophantine equation, the mean value in question counts the number of integral solutions of the equation

$$
y_{1}^{3}-y_{2}^{3}=z_{1}^{3}-z_{2}^{3}+z_{3}^{3}-z_{4}^{3}+z_{5}^{3}-z_{6}^{3},
$$

with

$$
Q<y_{1}, y_{2} \leq 2 Q \quad \text { and } \quad z_{i} \in \mathcal{A}(R)(1 \leq i \leq 6)
$$

When $y_{1}>y_{2}$, the left-hand side of this equation exceeds $3 Q^{2}$, whereas the right-hand side is always smaller than $3 R^{3}$. Since $R^{3} \leq Q^{2}$, we conclude that all solutions $\mathbf{y}, \mathbf{z}$ of (2.6) satisfy $y_{1}=y_{2}$, whence

$$
\int_{0}^{1}\left|g(\alpha)^{2} h(\alpha)^{6}\right| d \alpha \leq Q \int_{0}^{1}|h(\alpha)|^{6} d \alpha .
$$

The desired conclusion now follows from (2.2). 
Lemma 2.2. Suppose that $Q=P^{5 / 6}$ and that $P^{4 / 11} \leq R \leq P^{14 / 33-\tau / 6}$. Then one has

$$
T_{1} \ll P^{1+\varepsilon} Q R^{13 / 4-\tau} .
$$

Proof. By considering the diophantine equation underlying the mean value $(2.3)$, we find that $T_{1}$ counts the number of integral solutions of the equation

$$
x_{1}^{3}-x_{2}^{3}=y_{1}^{3}-y_{2}^{3}+z_{1}^{3}-z_{2}^{3}+z_{3}^{3}-z_{4}^{3}+z_{5}^{3}-z_{6}^{3},
$$

with $P<x_{1}, x_{2} \leq 2 P$, and $\mathbf{y}, \mathbf{z}$ as in (2.7). On making use of Lemma 2.1, one discerns that the diagonal contribution $I_{0}$, arising from those solutions of (2.8) counted by $T_{1}$ in which $x_{1}=x_{2}$, satisfies

$$
I_{0} \ll P Q R^{13 / 4-\tau}
$$

For the remaining solutions, it suffices by symmetry to consider the situation wherein $x_{1}>x_{2}$. On substituting $h=x_{1}-x_{2}$, one deduces from (2.8) that

$$
3 h P^{2}<(2 Q)^{3}+3 R^{3}<9 P^{5 / 2}
$$

whence $h<3 P^{1 / 2}$. Consequently, a consideration of the underlying diophantine equation reveals that the contribution of such solutions is at most

$$
I_{1}=\int_{0}^{1} \Psi(\alpha)\left|g(\alpha)^{2} h(\alpha)^{6}\right| d \alpha
$$

where

$$
\Psi(\alpha)=\sum_{1 \leq h<3 P^{1 / 2}} \sum_{P<x \leq 2 P} e\left(\alpha h\left(3 x^{2}+3 x h+h^{2}\right)\right) .
$$

We estimate the integral (2.10) by using the Hardy-Littlewood method. When $a \in \mathbb{Z}$ and $q \in \mathbb{N}$, define the auxiliary major arcs $\mathfrak{N}(q, a)$ by putting

$$
\mathfrak{N}(q, a)=\left\{\alpha \in[0,1):|q \alpha-a| \leq P Q^{-3}\right\},
$$

and then take $\mathfrak{N}$ to be the union of the $\operatorname{arcs} \mathfrak{N}(q, a)$ with $0 \leq a \leq q \leq P$ and $(a, q)=1$. Next, define $\Upsilon(\alpha)$ for $\alpha \in[0,1)$ by taking

$$
\Upsilon(\alpha)=\left(q+Q^{3}|q \alpha-a|\right)^{-1}
$$

when $\alpha \in \mathfrak{N}(q, a) \subseteq \mathfrak{N}$, and otherwise by putting $\Upsilon(\alpha)=0$. Then, as a consequence of the lemma in [10] combined with a standard transference principle 
(see $[14$, Section 2.8, Exercise 2]), one finds that whenever $a \in \mathbb{Z}$ and $q \in \mathbb{N}$ satisfy $(a, q)=1$ and $|\alpha-a / q| \leq q^{-2}$, one has

$$
\Psi(\alpha) \ll P^{3 / 2+\varepsilon}\left(\left(q+Q^{3}|q \alpha-a|\right)^{-1}+P^{-1}+\left(q+Q^{3}|q \alpha-a|\right) P^{-5 / 2}\right)^{1 / 2} .
$$

Hence, on applying Dirichlet's approximation theorem and recalling that $Q=P^{5 / 6}$, one swiftly arrives at the estimate

$$
\Psi(\alpha) \ll P^{3 / 2+\varepsilon} \Upsilon(\alpha)^{1 / 2}+P^{1+\varepsilon},
$$

valid uniformly for $\alpha \in[0,1)$. Substituting this upper bound into (2.10), and again applying Lemma 2.1, we conclude that

$$
I_{1} \ll P^{1+\varepsilon} Q R^{13 / 4-\tau}+P^{3 / 2+\varepsilon} I_{2},
$$

where

$$
I_{2}=\int_{0}^{1} \Upsilon(\alpha)^{1 / 2}\left|g(\alpha)^{2} h(\alpha)^{6}\right| d \alpha .
$$

Next, given a natural number $q$, we define $q_{1}$ to be the largest integer whose cube divides $q$, and we then put $q_{0}=q / q_{1}^{3}$. The function $\kappa(q)$ defined by taking $\kappa(q)=q_{0}^{-1 / 2} q_{1}^{-1}$ is multiplicative. For future use, we define the function $g^{+}(\alpha)$ for $\alpha \in[0,1)$ by putting

$$
g^{+}(\alpha)=q \kappa(q) Q \Upsilon(\alpha)
$$

when $\alpha \in \mathfrak{N}(q, a) \subseteq \mathfrak{N}$, and otherwise by taking $g^{+}(\alpha)=0$. On referring to [14, Theorem 4.1 and Lemmas 4.3, 4.4, 4.5, and 6.2], one readily confirms that the estimate

$$
g(\alpha) \ll P^{\varepsilon} g^{+}(\alpha)+P^{1 / 2+\varepsilon}
$$

holds uniformly for $\alpha \in \mathfrak{N}$. Substituting this bound into (2.12), we deduce that

$$
I_{2} \ll P^{1 / 2+\varepsilon} I_{3}+P^{\varepsilon} I_{4}
$$

where

$$
I_{3}=\int_{0}^{1} \Upsilon(\alpha)^{1 / 2}\left|g(\alpha) h(\alpha)^{6}\right| d \alpha
$$


and

$$
I_{4}=\int_{0}^{1} \Upsilon(\alpha)^{1 / 2}\left|g^{+}(\alpha) g(\alpha) h(\alpha)^{6}\right| d \alpha .
$$

Our investigation of $I_{3}$ begins with the application of [2, Lemma 2]. By hypothesis, we have $R \geq P^{4 / 11}$, and thus we are led via (2.2) to the bound

$$
\int_{0}^{1} \Upsilon(\alpha)|h(\alpha)|^{6} d \alpha \ll Q^{\varepsilon-3}\left(P \int_{0}^{1}|h(\alpha)|^{6} d \alpha+R^{6}\right) \ll Q^{\varepsilon-3} R^{6} .
$$

Applying Schwarz's inequality to (2.16), and then applying Lemma 2.1, we therefore see that

$$
\begin{aligned}
I_{3} & \leq\left(\int_{0}^{1} \Upsilon(\alpha)|h(\alpha)|^{6} d \alpha\right)^{1 / 2}\left(\int_{0}^{1}\left|g(\alpha)^{2} h(\alpha)^{6}\right| d \alpha\right)^{1 / 2} \\
& \ll\left(Q^{\varepsilon-3} R^{6}\right)^{1 / 2}\left(Q R^{13 / 4-\tau}\right)^{1 / 2}
\end{aligned}
$$

We turn our attention next to $I_{4}$. On recalling (2.12), a trivial estimate for $h(\alpha)$ in combination with an application of Hölder's inequality conveys us from (2.17) to the bound

$$
I_{4} \leq\left(R^{6} \int_{0}^{1} g^{+}(\alpha)^{4} d \alpha\right)^{1 / 4}\left(\int_{0}^{1} \Upsilon(\alpha)|h(\alpha)|^{6} d \alpha\right)^{1 / 4} I_{2}^{1 / 2} .
$$

In view of our definition (2.13), a routine computation confirms that

$$
\int_{0}^{1} g^{+}(\alpha)^{4} d \alpha \ll P^{\varepsilon} Q
$$

Consequently, on recalling (2.18), we obtain the upper bound

$$
I_{4} \ll P^{\varepsilon} Q^{-1 / 2} R^{3} I_{2}^{1 / 2} .
$$

Again making use of the hypothesis $R \geq P^{4 / 11}$, we therefore see from (2.15) and (2.19) that

$$
I_{2} \ll P^{1 / 2+\varepsilon} Q^{-1} R^{37 / 8-\tau / 2}+P^{\varepsilon} Q^{-1} R^{6} \ll P^{\varepsilon} Q^{-1} R^{6} .
$$

We substitute this estimate into (2.11) to obtain

$$
I_{1} \ll P^{1+\varepsilon} Q R^{13 / 4-\tau}+P^{3 / 2+\varepsilon} Q^{-1} R^{6} .
$$

A modest computation confirms that the first term here dominates under the hypothesis that $R \leq P^{14 / 33-\tau / 6}$, and thus the conclusion of the lemma follows by reference to $(2.9)$. 
We next supply an estimate for $T_{2}$ by combining routine diminishing ranges arguments.

Lemma 2.3. When $1 \leq Q \leq P$, one has

$$
T_{2} \ll P^{\varepsilon}\left(P Q^{2}+P^{-1} Q^{9 / 2}\right) .
$$

Moreover, provided that $P^{4 / 5} \leq Q \leq P$, one has

$$
T_{2} \ll P^{\varepsilon}\left(P Q^{2}+P^{-3 / 2} Q^{5}\right) .
$$

In particular, when $1 \leq Q \leq P^{5 / 6}$, one has $T_{2} \ll P^{1+\varepsilon} Q^{2}$.

Proof. Write $M=P Q^{-1}$ and $H=P M^{-3}$. Then the argument leading to $[10$, equation (4)] takes us from $(2.4)$ to the estimate

$$
T_{2} \ll P^{1+\varepsilon} Q^{2}+\int_{0}^{1} \Phi(\alpha)|g(\alpha)|^{4} d \alpha,
$$

where

$$
\Phi(\alpha)=\sum_{1 \leq h \leq 6 H} \sum_{P<x \leq 2 P} e\left(\alpha h\left(3 x^{2}+3 x h+h^{2}\right)\right) .
$$

A simple modification of the familiar proof of Hua's lemma (see, e.g., the argument of [16, page 438]) shows that

$$
\int_{0}^{1}|\Phi(\alpha)|^{4} d \alpha \ll H^{3} P^{2+\varepsilon}
$$

Thus, by applying Hölder's inequality in combination with Hua's lemma (see [14, Lemma 2.5]), we deduce that

$$
\begin{aligned}
T_{2} & \ll P^{1+\varepsilon} Q^{2}+\left(\int_{0}^{1}|\Phi(\alpha)|^{4} d \alpha\right)^{1 / 4}\left(\int_{0}^{1}|g(\alpha)|^{8} d \alpha\right)^{1 / 4}\left(\int_{0}^{1}|g(\alpha)|^{4} d \alpha\right)^{1 / 2} \\
& \ll P^{1+\varepsilon} Q^{2}+P^{\varepsilon}\left(H^{3} P^{2}\right)^{1 / 4}\left(Q^{5}\right)^{1 / 4}\left(Q^{2}\right)^{1 / 2} \\
& \ll P^{\varepsilon}\left(P Q^{2}+P^{-1} Q^{9 / 2}\right) .
\end{aligned}
$$

This confirms the first conclusion of the lemma.

Suppose next that $P^{4 / 5} \leq Q \leq P$. In this situation, the argument of the lemma in [10] shows that when $\alpha \in \mathbb{R}, a \in \mathbb{Z}$, and $q \in \mathbb{N}$ satisfy $|\alpha-a / q| \leq$ $q^{-2}$ and $(a, q)=1$, then

$$
\Phi(\alpha) \ll P^{\varepsilon}\left(H P q^{-1 / 2}+H P^{1 / 2}+H^{1 / 2} q^{1 / 2}\right) .
$$


Thus, following the argument of [10, pages 19, 20], we see that

$$
\begin{aligned}
T_{2} & \ll P^{1+\varepsilon} Q^{2}+P^{1 / 2+\varepsilon} H Q^{2}+P^{\varepsilon} \sum_{1 \leq q \leq P}\left(H P Q q^{-5 / 6}+P^{2} q^{-1 / 2}\right) \\
& \ll P^{1+\varepsilon} Q^{2}+P^{1 / 2+\varepsilon} H Q^{2}\left(P^{2 / 3} Q^{-1}+M^{5} P^{-1}+1\right) .
\end{aligned}
$$

When $P^{4 / 5} \leq Q \leq P$, we therefore conclude that

$$
T_{2} \ll P^{1+\varepsilon} Q^{2}+P^{1 / 2+\varepsilon} H Q^{2},
$$

an estimate that yields the second conclusion of the lemma.

The final assertion of the lemma follows from the first when $Q \leq P^{4 / 5}$, and from the second when $P^{4 / 5} \leq Q \leq P^{5 / 6}$.

We finish this section by swiftly disposing of the mean value $T_{3}$.

Lemma 2.4. Suppose that $R \leq Q \leq P^{5 / 6}$. Then one has

$$
T_{3} \ll P^{1+\varepsilon} Q R
$$

Proof. Since $R \leq Q \leq P^{5 / 6}$, if we first apply Schwarz's inequality to (2.5) and then make use of the final estimate of Lemma 2.3, we obtain

$$
\begin{aligned}
T_{3} & \leq\left(\int_{0}^{1}\left|f(\alpha)^{2} g(\alpha)^{4}\right| d \alpha\right)^{1 / 2}\left(\int_{0}^{1}\left|f(\alpha)^{2} h(\alpha)^{4}\right| d \alpha\right)^{1 / 2} \\
& \ll P^{\varepsilon}\left(P Q^{2}\right)^{1 / 2}\left(P R^{2}\right)^{1 / 2} .
\end{aligned}
$$

The desired conclusion follows.

\section{$\S 3$. An auxiliary minor arc estimate}

An active ingredient in our argument is an auxiliary minor arc estimate derived by analytic differencing. This section is devoted to the proof of an estimate for a certain eighth moment of cubic exponential sums that has arisen in a weaker form in the earlier work of Vaughan [13]. We must first introduce some notation. Let $P$ be a large real number, and let $Y$ be a real number with $P^{1 / 8} \leq Y \leq P^{1 / 7}$. Also, when $X$ and $Z$ are positive numbers, define

$$
\mathcal{A}^{*}(X, Z)=\left\{n \in \mathbb{Z} \cap[1, X]: \varpi \text { prime and } \varpi \mid n \Rightarrow \varpi \leq Z^{\eta}\right\},
$$


and put $\mathcal{B}(X, Z)=\mathcal{A}^{*}(2 X, Z) \backslash \mathcal{A}^{*}(X, Z)$. Note that $\mathcal{A}(X)=\mathcal{A}^{*}(X, X)$. Define the exponential sums $k_{p}(\alpha)=k_{p}(\alpha ; Y)$ and $K(\alpha)=K(\alpha ; Y)$ by

$$
k_{p}(\alpha ; Y)=\sum_{w \in \mathcal{B}(P / p, 2 P / Y)} e\left(\alpha w^{3}\right) \quad \text { and } \quad K(\alpha ; Y)=\sum_{Y<p \leq 2 Y} k_{p}\left(p^{3} \alpha ; Y\right) \text {. }
$$

Also, write

$$
f_{p}(\alpha)=\sum_{\substack{P<x \leq 2 P \\(x, p)=1}} e\left(\alpha x^{3}\right) \quad \text { and } \quad l_{p}(\alpha)=\sum_{P / p<u \leq 2 P / p} e\left(\alpha u^{3}\right)
$$

We next introduce a set of major and minor arcs suitable for our analysis. When $X$ is a real parameter with $1 \leq X \leq P^{3 / 2}$, we define

$$
\mathfrak{M}(q, a ; X)=\left\{\alpha \in[0,1):|q \alpha-a| \leq X P^{-3}\right\}
$$

and we then take $\mathfrak{M}(X)$ to be the union of the $\operatorname{arcs} \mathfrak{M}(q, a ; X)$ with $0 \leq a \leq$ $q \leq X$ and $(a, q)=1$. We then put $\mathfrak{m}(X)=[0,1) \backslash \mathfrak{M}(X)$.

The key theorem of this section provides a bound for the eighth moment

$$
\mathfrak{T}(Y)=\int_{\mathfrak{m}\left(P Y^{3}\right)}\left|f(\alpha)^{2} K(\alpha)^{6}\right| d \alpha
$$

THEOREM 3.1. Whenever $P^{1 / 8} \leq Y \leq P^{1 / 7}$, one has

$$
\mathfrak{T}(Y) \ll P^{19 / 4} Y^{-3 / 4}(P / Y)^{\varepsilon-\tau}+P^{9 / 2+\varepsilon} Y .
$$

We note that Vaughan [13] has analyzed the mean value (3.3) in the special case $Y=P^{1 / 8}$. In this restricted situation, the antepenultimate display of [13, Section 5] supplies a bound similar to that given by Theorem 3.1, though weaker by a factor exceeding $Y^{1 / 2}$. The estimate supplied by our theorem matches in strength the bounds made available, by the interwoven arrangements of generating functions, applied in the arguments leading to the sharpest available estimates for the exceptional set in Waring's problem for sums of four cubes (see [4]). The latter arguments fail to capture the expected number of solutions, since the generating functions applied do not have full density, a deficiency that is absent from the bound summarized below in Corollary 3.2. 
Proof. Our argument involves a careful consideration of the possible prime factors implicit in the generating functions $K(\alpha)$ that are common to the exponential sums $f(\alpha)$ lying within the mean value in question. It is convenient throughout to write $\mathfrak{m}=\mathfrak{m}\left(P Y^{3}\right)$. Furthermore, within this proof, summations over the variable $p$ will denote sums over prime numbers $p$ with $p \equiv 2(\bmod 3)$ and $Y<p \leq 2 Y$.

On recalling (3.2), we see that for any prime number $p$, one has

$$
f(\alpha)=f_{p}(\alpha)+l_{p}\left(p^{3} \alpha\right)
$$

Consequently, it follows from (3.1) that

$$
\begin{aligned}
f(\alpha)^{2} K(\alpha) & =\sum_{p} f(\alpha)^{2} k_{p}\left(p^{3} \alpha\right) \\
& \ll \sum_{p}\left(\left|f_{p}(\alpha)^{2} k_{p}\left(p^{3} \alpha\right)\right|+\left|l_{p}\left(p^{3} \alpha\right)^{2} k_{p}\left(p^{3} \alpha\right)\right|\right) .
\end{aligned}
$$

On substituting the latter relation into (3.3), we find that

$$
\mathfrak{T}(Y) \ll \mathfrak{T}_{1}+\mathfrak{T}_{2},
$$

where

$$
\mathfrak{T}_{1}=\int_{\mathfrak{m}}|K(\alpha)|^{5} \sum_{p}\left|f_{p}(\alpha)^{2} k_{p}\left(p^{3} \alpha\right)\right| d \alpha
$$

and

$$
\mathfrak{T}_{2}=\int_{\mathfrak{m}}|K(\alpha)|^{5} \sum_{p}\left|l_{p}\left(p^{3} \alpha\right)^{2} k_{p}\left(p^{3} \alpha\right)\right| d \alpha .
$$

An application of Hölder's inequality reveals that

$$
\sum_{p}\left|f_{p}(\alpha)^{2} k_{p}\left(p^{3} \alpha\right)\right| \leq\left(\sum_{p}\left|f_{p}(\alpha)^{2} k_{p}\left(p^{3} \alpha\right)^{6}\right|\right)^{1 / 6}\left(\sum_{p}\left|f_{p}(\alpha)\right|^{2}\right)^{5 / 6} .
$$

Applying Hölder's inequality a second time, we derive from (3.6) the bound

$$
\mathfrak{T}_{1} \leq \mathfrak{T}_{3}^{1 / 6} \mathfrak{T}_{4}^{5 / 6}
$$

where

$$
\mathfrak{T}_{3}=\sum_{p} \int_{\mathfrak{m}}\left|f_{p}(\alpha)^{2} k_{p}\left(p^{3} \alpha\right)^{6}\right| d \alpha
$$


and

$$
\mathfrak{T}_{4}=\int_{\mathfrak{m}}|K(\alpha)|^{6} \sum_{p}\left|f_{p}(\alpha)\right|^{2} d \alpha .
$$

We return to the consideration of $\mathfrak{T}_{3}$ later in the proof, for this is the central object of our attention. So far as $\mathfrak{T}_{4}$ is concerned, we may make use again of (3.4) to deduce that

$$
\mathfrak{T}_{4} \ll \mathfrak{T}_{5}+\mathfrak{T}_{6},
$$

where

$$
\mathfrak{T}_{5}=\sum_{p} \int_{\mathfrak{m}}\left|f(\alpha)^{2} K(\alpha)^{6}\right| d \alpha
$$

and

$$
\mathfrak{T}_{6}=\int_{\mathfrak{m}}|K(\alpha)|^{6} \sum_{p}\left|l_{p}\left(p^{3} \alpha\right)\right|^{2} d \alpha .
$$

In view of $(3.3)$, one has $\mathfrak{T}_{5} \ll Y \mathfrak{T}(Y)$. In addition, when $Y<p \leq 2 Y$, the discussion surrounding $[13$, equation (34)] supplies the estimate

$$
\sup _{\alpha \in \mathfrak{m}}\left|l_{p}\left(p^{3} \alpha\right)\right| \ll(P / p)^{3 / 4+\varepsilon},
$$

and, on considering the underlying diophantine equations, [5, Lemma 2] delivers the bound

$$
\int_{0}^{1}|K(\alpha)|^{6} d \alpha \ll P^{3+\varepsilon} Y^{2} .
$$

We therefore deduce from (3.11) that

$$
\mathfrak{T}_{6} \ll P^{3 / 2+\varepsilon} Y^{-1 / 2} \int_{0}^{1}|K(\alpha)|^{6} d \alpha \ll P^{9 / 2+\varepsilon} Y^{3 / 2} .
$$

Thus, on substituting this together with our earlier estimate for $\mathfrak{T}_{5}$ into (3.10), we obtain the upper bound

$$
\mathfrak{T}_{4} \ll Y\left(\mathfrak{T}(Y)+P^{9 / 2+\varepsilon} Y^{1 / 2}\right) .
$$

We turn our attention next to $\mathfrak{T}_{2}$, first applying Cauchy's inequality to obtain

$$
\sum_{p}\left|l_{p}\left(p^{3} \alpha\right)^{2} k_{p}\left(p^{3} \alpha\right)\right| \leq\left(\sum_{p}\left|l_{p}\left(p^{3} \alpha\right)\right|^{4}\right)^{1 / 2}\left(\sum_{p}\left|k_{p}\left(p^{3} \alpha\right)\right|^{2}\right)^{1 / 2} .
$$


Substituting into (3.7) and recalling (3.12), we discover that

$$
\mathfrak{T}_{2} \ll P^{3 / 2+\varepsilon} Y^{-1} \int_{0}^{1}|K(\alpha)|^{5}\left(\sum_{p}\left|k_{p}\left(p^{3} \alpha\right)\right|^{2}\right)^{1 / 2} d \alpha .
$$

An application of Hölder's inequality therefore reveals that

$$
\mathfrak{T}_{2} \ll P^{3 / 2+\varepsilon} Y^{-1}\left(\int_{0}^{1}|K(\alpha)|^{6} d \alpha\right)^{1 / 2} \mathfrak{T}_{7}^{1 / 2},
$$

where

$$
\mathfrak{T}_{7}=\int_{0}^{1}|K(\alpha)|^{4} \sum_{p}\left|k_{p}\left(p^{3} \alpha\right)\right|^{2} d \alpha .
$$

On considering the underlying diophantine equations, one sees that

$$
\int_{0}^{1}|K(\alpha)|^{4} \sum_{p}\left|k_{p}\left(p^{3} \alpha\right)\right|^{2} d \alpha \ll \int_{0}^{1}|K(\alpha)|^{6} d \alpha
$$

and hence it follows from (3.13) that

$$
\mathfrak{T}_{2} \ll P^{\varepsilon}\left(P^{3 / 2} Y^{-1}\right)\left(P^{3} Y^{2}\right)=P^{9 / 2+\varepsilon} Y .
$$

We now substitute (3.8), (3.14), and (3.15) into (3.5) to obtain the upper bound

$$
\mathfrak{T}(Y) \ll P^{9 / 2+\varepsilon} Y+\left(Y^{5} \mathfrak{T}_{3}\right)^{1 / 6} \mathfrak{T}(Y)^{5 / 6}+\left(Y^{5} \mathfrak{T}_{3}\right)^{1 / 6}\left(P^{9 / 2+\varepsilon} Y^{1 / 2}\right)^{5 / 6} .
$$

We therefore have

$$
\begin{aligned}
\mathfrak{T}(Y) & \ll P^{9 / 2+\varepsilon} Y+Y^{5} \mathfrak{T}_{3}+\left(Y^{5} \mathfrak{T}_{3}\right)^{1 / 6}\left(P^{9 / 2+\varepsilon} Y^{1 / 2}\right)^{5 / 6} \\
& \ll P^{9 / 2+\varepsilon} Y+Y^{5} \mathfrak{T}_{3} .
\end{aligned}
$$

Write

$$
k^{*}(\alpha)=\max _{Y<p \leq 2 Y}\left|k_{p}(\alpha)\right|
$$

Then by applying [5, Lemma 4], we deduce from (3.9) that

$$
\mathfrak{T}_{3} \ll P^{3 / 2+\varepsilon} Y^{-5 / 2} \int_{0}^{1} k^{*}(\alpha)^{6} d \alpha .
$$


The Carleson-Hunt theorem (see [8, Theorem 1]), in combination with (2.2) above, shows that

$$
\int_{0}^{1} k^{*}(\alpha)^{6} d \alpha \ll \int_{0}^{1}\left|\sum_{w \in \mathcal{A}(2 P / Y)} e\left(\alpha w^{3}\right)\right|^{6} d \alpha \ll(P / Y)^{13 / 4-\tau},
$$

and thus we see that

$$
\mathfrak{T}_{3} \ll P^{19 / 4} Y^{-23 / 4}(P / Y)^{\varepsilon-\tau} .
$$

The proof of Theorem 3.1 is completed by substituting this estimate into (3.16).

It is convenient to have available a variant of Theorem 3.1 that facilitates simplifications in associated major arc analyses. We first introduce some additional notation. Put $J=[(1 / 2) \tau \log P]$, and define the exponential sum

$$
\widetilde{K}(\alpha)=\sum_{j=1}^{J} K\left(\alpha ; 2^{-j} Y\right)
$$

and the mean value

$$
\widetilde{\mathfrak{T}}(Y)=\int_{\mathfrak{m}\left(P Y^{3}\right)}\left|f(\alpha)^{2} \widetilde{K}(\alpha)^{6}\right| d \alpha .
$$

COROLlaRY 3.2. Whenever $P^{1 / 8+\tau / 2} \leq Y \leq P^{1 / 7}$, one has

$$
\widetilde{\mathfrak{T}}(Y) \ll P^{19 / 4-\tau / 2} Y^{-3 / 4}+P^{9 / 2+\varepsilon} Y .
$$

Proof. An application of Hölder's inequality leads from (3.18) to the bound

$$
\int_{\mathfrak{m}\left(P Y^{3}\right)}\left|f(\alpha)^{2} \widetilde{K}(\alpha)^{6}\right| d \alpha \ll(\log P)^{6} \max _{1 \leq j \leq J} \mathfrak{T}\left(2^{-j} Y\right) .
$$

The desired conclusion now follows from Theorem 3.1 with a modicum of computation.

\section{§4. The application of the circle method}

Having equipped ourselves with the tools required in our application of the Hardy-Littlewood method, our goal in this section is to engineer the framework required for the application of Bessel's inequality to the problem 
of estimating the exceptional set at hand. Let $N$ be a large positive number, and write

$$
P=(N / 4)^{1 / 3}, \quad Q=P^{5 / 6}, \quad Y=P^{11 / 79}, \quad \text { and } \quad L=(\log P)^{1 / 10} .
$$

We consider a parameter $\theta$ with $0 \leq \theta \leq 1 / 3$, write $\phi=3 \theta$, and fix $R=P^{\phi}$. In addition, we put $J=[(1 / 2) \tau \log P]$, as in the previous section. Define $\rho(n)=\rho_{\theta}(n)$ to be the number of integral representations of $n$ in the form

$$
n=x^{3}+(p w)^{3}+y^{3}+z^{3}
$$

with

$$
\begin{gathered}
P<x \leq 2 P, \quad Q<y \leq 2 Q, \quad z \in \mathcal{A}(R), \\
2^{-j} Y<p \leq 2^{1-j} Y, \quad \text { and } \quad w \in \mathcal{B}\left(P / p, 2^{1+j} P / Y\right) \quad(1 \leq j \leq J) .
\end{gathered}
$$

Our goal is to establish that, when $362 / 869 \leq \phi \leq 14 / 33-\tau / 6$, then for almost all values of $n$ with $N<n \leq 2 N$, one has $\rho_{\theta}(n) \gg n^{\theta-1 / 18}$. Since $r_{\theta}(n) \geq \rho_{\theta}(n)$, the conclusion of Theorem 1.1 follows by summing over dyadic intervals.

Next, given a measurable set $\mathfrak{B} \subseteq[0,1)$, we define

$$
\rho(n ; \mathfrak{B})=\int_{\mathfrak{B}} f(\alpha) \widetilde{K}(\alpha) g(\alpha) h(\alpha) e(-n \alpha) d \alpha .
$$

By orthogonality, one then has $\rho_{\theta}(n)=\rho(n ;[0,1))$. We estimate the latter quantity by means of the circle method. Our argument involves two pruning steps, and we therefore introduce various classes of arcs to facilitate the analysis. First, when $a \in \mathbb{Z}, q \in \mathbb{N}$, and $(a, q)=1$, we put

$$
\mathfrak{P}(q, a)=\left\{\alpha \in[0,1):|\alpha-a / q| \leq L N^{-1}\right\} .
$$

We then define $\mathfrak{P}$ to be the union of the $\operatorname{arcs} \mathfrak{P}(q, a)$ with $0 \leq a \leq q \leq L$ and $(a, q)=1$, and we write $\mathfrak{p}=[0,1) \backslash \mathfrak{P}$. Next, in the notation introduced in the previous section prior to the statement of Theorem 3.1 , when $1 \leq X \leq P^{3 / 2}$, we define $\mathfrak{K}(X)=\mathfrak{M}(2 X) \backslash \mathfrak{M}(X)$. In addition, we write

$\mathfrak{m}=\mathfrak{m}\left(P Y^{3}\right), \quad \mathfrak{V}=\mathfrak{M}\left(P Y^{3}\right) \backslash \mathfrak{M}\left(P^{6 / 5}\right), \quad$ and $\quad \mathfrak{U}=\mathfrak{M}\left(P^{6 / 5}\right) \backslash \mathfrak{P}$.

Finally, it is convenient for future reference to introduce the generating functions

$$
S(q, a)=\sum_{r=1}^{q} e\left(a r^{3} / q\right) \quad \text { and } \quad v(\beta)=\int_{P}^{2 P} e\left(\beta \gamma^{3}\right) d \gamma .
$$


LEMma 4.1. One has $\rho(n ; \mathfrak{P}) \gg n^{\theta-1 / 18}$ for all integers $n$ satisfying $N<$ $n \leq 2 N$, with at most $O\left(N L^{-1 / 16}\right)$ possible exceptions.

Proof. By [14, Theorem 4.1], when $a \in \mathbb{Z}, q \in \mathbb{N}$, and $\beta \in \mathbb{R}$, one has

$$
f(\beta+a / q)-q^{-1} S(q, a) v(\beta) \ll q^{1 / 2+\varepsilon}\left(1+P^{3}|\beta|\right)^{1 / 2} .
$$

Hence, when $\alpha \in \mathfrak{P}(q, a) \subseteq \mathfrak{P}$, one sees that

$$
f(\alpha)-q^{-1} S(q, a) v(\alpha-a / q) \ll L^{1+\varepsilon} .
$$

Also, on examining the Taylor expansion of $e\left(\beta \gamma^{3}\right)$, one finds in like manner that when $\alpha \in \mathfrak{P}(q, a) \subseteq \mathfrak{P}$, then

$$
g(\alpha)-q^{-1} S(q, a) g(0) \ll L Q^{4} P^{-3} \ll Q^{1 / 2} .
$$

Similarly, it follows from $[15$, Lemma 8.5] that for $\alpha \in \mathfrak{P}(q, a) \subseteq \mathfrak{P}$, one has

$$
h(\alpha)-q^{-1} S(q, a) h(0) \ll R L^{-5},
$$

and that when $1 \leq j \leq J$ and $2^{-j} Y<p \leq 2^{1-j} Y$, there exists a positive number $c$, depending only on $\eta$, such that

$$
k_{p}\left(p^{3} \alpha ; 2^{-j} Y\right)-c q^{-1} S\left(q, a p^{3}\right) \int_{P / p}^{2 P / p} e\left(p^{3} \gamma^{3}(\alpha-a / q)\right) d \gamma \ll P p^{-1} L^{-5} .
$$

We note that when $2^{-J} Y<p \leq Y$ and $q \leq L$, one has $p \nmid q$, and so one may apply a change of variables to show that $S\left(q, a p^{3}\right)=S(q, a)$. Following another change of variables, one sees that

$$
\widetilde{K}(\alpha)-\sum_{2^{-J} Y<p \leq Y} c(p q)^{-1} S(q, a) v(\alpha-a / q) \ll P L^{-5} .
$$

The prime number theorem in arithmetic progressions implies that

$$
\sum_{2^{-J} Y<p \leq Y} p^{-1}=\frac{1}{2} \log \left(\frac{\log Y}{\log \left(2^{-J} Y\right)}\right)+O\left(\frac{1}{\log Y}\right),
$$

and thus we deduce that

$$
\widetilde{K}(\alpha)-C q^{-1} S(q, a) v(\alpha-a / q) \ll P L^{-5},
$$


where

$$
C=\frac{c}{2} \log \left(\frac{22}{22-(79 \log 2) \tau}\right)>0
$$

Next, write

$$
T(q, a)=q^{-4} S(q, a)^{4} \quad \text { and } \quad u(\beta)=C v(\beta)^{2} g(0) h(0) .
$$

Then, from the above approximations, one discerns that for $\alpha \in \mathfrak{P}(q, a) \subseteq \mathfrak{P}$, one has

$$
f(\alpha) \widetilde{K}(\alpha) g(\alpha) h(\alpha)-T(q, a) u(\alpha-a / q) \ll P^{2} Q R L^{-5} .
$$

Since the measure of $\mathfrak{P}$ is $O\left(L^{3} N^{-1}\right)$, from (4.1) we reach the formula

$$
\rho(n ; \mathfrak{P})=\mathfrak{S}(n ; L) \mathcal{J}(n ; L)+O\left(P^{2} Q R N^{-1} L^{-2}\right),
$$

where

$$
\mathfrak{S}(n ; L)=\sum_{1 \leq q \leq L} A(q, n),
$$

in which we have written

$$
A(q, n)=\sum_{\substack{a=1 \\(a, q)=1}}^{q} T(q, a) e(-n a / q)
$$

and

$$
\mathcal{J}(n ; L)=\int_{-L / N}^{L / N} u(\beta) e(-\beta n) d \beta .
$$

The expression $\mathfrak{S}(n ; L)$ is a partial sum of the familiar singular series

$$
\mathfrak{S}(n)=\sum_{q=1}^{\infty} A(q, n)
$$

associated with sums of four cubes. The standard theory of singular series establishes that the series $\mathfrak{S}(n)$ converges absolutely and satisfies $1 \ll \mathfrak{S}(n) \ll$ $(\log \log n)^{4}($ see [14, Theorem 4.3] and [9, equation (1.3)]). Moreover, on recalling the notation introduced prior to $(2.13)$, we find from [14, Lemmas 2.11 and 4.7] that $A(q, n) \ll q^{1 / 2+\varepsilon} \kappa(q)^{4}(q, n)$. We therefore deduce that

$$
\sum_{N<n \leq 2 N}|\mathfrak{S}(n)-\mathfrak{S}(n ; L)| \leq \sum_{N<n \leq 2 N} \sum_{q=1}^{\infty}(q / L)^{1 / 8}|A(q, n)|
$$




$$
\begin{aligned}
& \ll L^{-1 / 8} \sum_{q=1}^{\infty} q^{2 / 3} \kappa(q)^{4} \sum_{d \mid q} \sum_{\substack{N<n \leq 2 N \\
(q, n)=d}}(q, n) \\
& \ll N L^{-1 / 8} \sum_{q=1}^{\infty} q^{2 / 3} \kappa(q)^{4} d(q),
\end{aligned}
$$

where $d(q)$ denotes the number of distinct divisors of $q$. The final sum over $q$ converges, since

$$
\sum_{q=1}^{\infty} q^{2 / 3} \kappa(q)^{4} d(q)=\prod_{\varpi \text { prime }}\left(1+2 \varpi^{-4 / 3}+O\left(\varpi^{-2}\right)\right) \ll 1,
$$

and hence the inequality $|\mathfrak{S}(n)-\mathfrak{S}(n ; L)|>L^{-1 / 16}$ can hold for at most $O\left(N L^{-1 / 16}\right)$ integers $n$ with $N<n \leq 2 N$. In particular, one may conclude that $|\mathfrak{S}(n ; L)| \gg 1$ for all integers $n$ satisfying $N<n \leq 2 N$, with at most $O\left(N L^{-1 / 16}\right)$ possible exceptions.

Integration by parts, meanwhile, confirms the estimate

$$
v(\beta) \ll P\left(1+P^{3}|\beta|\right)^{-1},
$$

and hence one has

$$
u(\beta) \ll P^{2} Q R\left(1+P^{3}|\beta|\right)^{-2} .
$$

It follows that

$$
\mathcal{J}(n ; L)-\int_{-\infty}^{\infty} u(\beta) e(-\beta n) d \beta \ll P^{2} Q R N^{-1} L^{-1} .
$$

The last integral here converges absolutely, in view of (4.9). It may therefore be evaluated by following a standard treatment, such as that described in $[7$, pages 21,22$]$. The result is that when $N<n \leq 2 N$, the integral is bounded below by $c_{0} P^{2} Q R N^{-1}$, where $c_{0}$ is a certain positive absolute constant. For the same values of $n$, it follows that

$$
\mathcal{J}(n ; L) \gg P^{2} Q R N^{-1} \text {. }
$$

On collecting these lower bounds within (4.5), we conclude that one has

$$
\rho(n ; \mathfrak{P}) \gg P^{2} Q R N^{-1} \gg n^{\theta-1 / 18}
$$

for all integers $n$ satisfying $N<n \leq 2 N$, with at most $O\left(N L^{-1 / 16}\right)$ possible exceptions. This completes the proof of the lemma. 
In Section 5 below, we show that when $362 / 869 \leq \phi \leq 14 / 33-\tau / 6$, then

$$
\sum_{N<n \leq 2 N}|\rho(n ; \mathfrak{p})|^{2} \ll N^{2 \theta+8 / 9} L^{-1 / 8},
$$

so that $|\rho(n ; \mathfrak{p})| \ll n^{\theta-1 / 18}(\log n)^{-1 / 200}$ for almost all natural numbers $n$ with $N<n \leq 2 N$. Granted this conclusion, it follows from Lemma 4.1 that for almost all such $n$, one has

$$
\rho_{\theta}(n)=\rho(n ; \mathfrak{P})+\rho(n ; \mathfrak{p}) \gg n^{\theta-1 / 18} .
$$

The conclusion of Theorem 1.1 follows on recalling that $r_{\theta}(n) \geq \rho_{\theta}(n)$ and then summing over dyadic intervals.

\section{$\S 5$. The minor arc contribution}

We derive (4.10) by applying Bessel's inequality, although several pruning operations are required.

Lemma 5.1. Provided that $362 / 869 \leq \phi \leq 14 / 33-\tau / 6$, one has

$$
\sum_{N<n \leq 2 N}|\rho(n ; \mathfrak{m})|^{2} \ll N^{2 \theta+8 / 9} L^{-10}
$$

Proof. By Bessel's inequality, it follows from (4.1) that

$$
\sum_{N<n \leq 2 N}|\rho(n ; \mathfrak{m})|^{2} \leq \int_{\mathfrak{m}}|f(\alpha) \widetilde{K}(\alpha) g(\alpha) h(\alpha)|^{2} d \alpha .
$$

An application of Hölder's inequality therefore yields the upper bound

$$
\sum_{N<n \leq 2 N}|\rho(n ; \mathfrak{m})|^{2} \leq T_{1}^{1 / 3} T_{2}^{1 / 3} \widetilde{\mathfrak{T}}(Y)^{1 / 3},
$$

where $T_{1}, T_{2}$, and $\widetilde{\mathfrak{T}}(Y)$ are respectively given by $(2.3),(2.4)$, and (3.19). Hence, by appealing to Lemmas 2.2 and 2.3 and Corollary 3.2 , we arrive at the estimate

$$
\begin{aligned}
\sum_{N<n \leq 2 N}|\rho(n ; \mathfrak{m})|^{2} & \ll P^{\varepsilon}\left(P Q R^{13 / 4-\tau}\right)^{1 / 3}\left(P Q^{2}\right)^{1 / 3}\left(P^{19 / 4-\tau / 2} Y^{-3 / 4}\right)^{1 / 3} \\
& \ll P^{9 / 4-\tau / 6+\varepsilon} Q R^{13 / 12-\tau / 3} Y^{-1 / 4}
\end{aligned}
$$


A modest computation now reveals that the conclusion of the lemma holds provided that

$$
P^{37 / 12} R^{13 / 12} Y^{-1 / 4} \leq P^{8 / 3} R^{2}
$$

as is guaranteed whenever $R \geq P^{5 / 11} Y^{-3 / 11}$. Consequently, whenever $\phi \geq$ $5 / 11-3 / 79=362 / 869$, the conclusion of the lemma follows.

We now come to the first pruning step.

Lemma 5.2. Provided that $362 / 869 \leq \phi \leq 14 / 33-\tau / 6$, one has

$$
\sum_{N<n \leq 2 N}|\rho(n ; \mathfrak{V})|^{2} \ll N^{2 \theta+8 / 9} L^{-10} .
$$

Proof. We begin by estimating the contribution arising from the set $\mathfrak{K}(X)$. By Bessel's inequality, it follows from (4.1) that when $1 \leq X \leq P Y^{3}$, one has

$$
\sum_{N<n \leq 2 N}|\rho(n ; \mathfrak{K}(X))|^{2} \leq \mathcal{I}_{0}
$$

where

$$
\mathcal{I}_{0}=\int_{\mathfrak{K}(X)}|f(\alpha) \widetilde{K}(\alpha) g(\alpha) h(\alpha)|^{2} d \alpha .
$$

When $X \geq P^{6 / 5}$, it is a consequence of (4.3), together with (4.8) and [14, Theorem 4.2], that

$$
\sup _{\alpha \in \mathfrak{K}(X)}|f(\alpha)| \ll P X^{-1 / 3}+X^{1 / 2+\varepsilon} \ll X^{1 / 2+\varepsilon} .
$$

Under the same hypotheses on $X$, therefore, an application of Hölder's inequality leads from (5.2) to the estimate

$$
\begin{aligned}
\mathcal{I}_{0} & \ll X^{1 / 6+\varepsilon} \int_{\mathfrak{K}(X)}|f(\alpha)|^{5 / 3}|\widetilde{K}(\alpha) g(\alpha) h(\alpha)|^{2} d \alpha \\
& \leq T_{1}^{1 / 3} T_{2}^{1 / 6}\left(X^{1 / 2+\varepsilon} \mathcal{I}_{1}\right)^{1 / 3}\left(\int_{0}^{1}\left|\widetilde{K}(\alpha)^{2} g(\alpha)^{4}\right| d \alpha\right)^{1 / 6}
\end{aligned}
$$

where $T_{1}$ and $T_{2}$ are given by (2.3) and (2.4), and

$$
\mathcal{I}_{1}=\int_{\mathfrak{K}(X)}\left|f(\alpha)^{2} \widetilde{K}(\alpha)^{5}\right| d \alpha .
$$


But on considering the diophantine equation underlying (2.4), and noting (2.1), (3.1), and (3.18), we find that

$$
T_{2} \geq \int_{0}^{1}\left|\widetilde{K}(\alpha)^{2} g(\alpha)^{4}\right| d \alpha
$$

and so (5.3) becomes

$$
\mathcal{I}_{0} \ll T_{1}^{1 / 3} T_{2}^{1 / 3}\left(X^{1 / 2+\varepsilon} \mathcal{I}_{1}\right)^{1 / 3} .
$$

The expression $\mathcal{I}_{1}$ has been examined already in the course of the proof of [5, Theorem 3], although in that treatment the focus is on the situation wherein $X=P Y^{3}$. A careful examination of that argument reveals that whenever $P^{6 / 5} \leq X \leq P Y^{3}$, one has the estimate

$$
\int_{\mathfrak{K}(X)}\left|f(\alpha)^{2} K(\alpha)^{5}\right| d \alpha \ll P^{4+\lambda} Y^{-1-\lambda}\left(P Y^{3} / X\right)^{1 / 2},
$$

in which we have written $\lambda=3 / 34-\tau / 4$. The validity of this claim requires a few words of justification. First, the estimates in [5, pages 28, 29] remain valid provided only that $X>8 Y^{3}(P / Y)^{3 / 4}$, as is guaranteed whenever $X \geq$ $P^{6 / 5}$ and $Y \leq P^{1 / 7}$ (see the discussion prior to [5, equation (5.10)]). Thus, the estimate (5.6) follows from a satisfactory modification of the proof of [5, Lemma 4], and in this proof the set $\mathfrak{n}$ now becomes the set of $\alpha \in[0,1)$ with the property that whenever $q$ is a natural number with $\|q \alpha\| \leq X Y^{-3} Q^{-3}$, then one has $q>X Y^{-3}$. In this lemma, we put $S(\alpha)=k^{*}(\alpha)^{5}$, with $k^{*}(\alpha)$ defined as in (3.17). Now, in the notation of [5], one finds at the top of page 27 that the estimate

$$
\sup _{\alpha \in \mathfrak{n}}\left(\sum_{1 \leq h \leq H}|F(\alpha h, \gamma ; h)|^{2}\right) \ll H P^{1+\varepsilon}\left(P Y^{3} / X\right)
$$

holds uniformly in $\gamma$. Likewise, provided that $X \geq Y^{6}$, one finds that

$$
\sup _{\alpha \in \mathfrak{n}}\left(\sum_{1 \leq h \leq H}\left|G_{h}\left(\alpha h^{3}, \pm \gamma h\right)\right|^{2}\right) \ll P^{\varepsilon} H Y .
$$

Thus, on checking the argument in [11, pages 155 and 156], one concludes from the argument of the proof of [5, Lemma 4] together with [5, Section 5] that

$$
\int_{\mathfrak{K}(X)}\left|f(\alpha)^{2} K(\alpha)^{5}\right| d \alpha \ll P^{3 / 2+\varepsilon} Y^{3 / 2}\left(P Y^{3} / X\right)^{1 / 2} \int_{0}^{1} k^{*}(\alpha)^{5} d \alpha .
$$


An application of the Carleson-Hunt theorem [8] in combination with [17, Theorem 1.2] reveals that

$$
\int_{0}^{1} k^{*}(\alpha)^{5} d \alpha \ll \int_{0}^{1}\left|\sum_{w \in \mathcal{A}(2 P / Y)} e\left(\alpha w^{3}\right)\right|^{5} d \alpha \ll(P / Y)^{44 / 17-2 \tau / 7},
$$

and the conclusion (5.6) follows at once.

An application of Hölder's inequality establishes that

$$
|\widetilde{K}(\alpha)|^{5} \ll(\log P)^{5} \max _{1 \leq j \leq J}\left|K\left(\alpha ; 2^{-j} Y\right)\right|^{5},
$$

and so we deduce from (5.4) and (5.6) that

$$
\begin{aligned}
\mathcal{I}_{1} & \ll(\log P)^{5} \max _{1 \leq j \leq J} \int_{\mathfrak{K}(X)}\left|f(\alpha)^{2} K\left(\alpha ; 2^{-j} Y\right)^{5}\right| d \alpha \\
& \ll P^{4+\lambda+\varepsilon} Y^{-1-\lambda}\left(P Y^{3} / X\right)^{1 / 2} .
\end{aligned}
$$

Substituting (5.7) into (5.5) along with the conclusions of Lemmas 2.2 and 2.3 , we find that

$$
\mathcal{I}_{0} \ll P^{\varepsilon}\left(P Q R^{13 / 4-\tau}\right)^{1 / 3}\left(P Q^{2}\right)^{1 / 3}\left(P^{9 / 2+\lambda} Y^{1 / 2-\lambda}\right)^{1 / 3} .
$$

Therefore, provided that $P^{6 / 5} \leq X \leq P Y^{3}$, we deduce that

$$
\mathcal{I}_{0} \ll P^{13 / 6+\lambda / 3+\varepsilon} Q R^{13 / 12-\tau / 3} Y^{1 / 6-\lambda / 3} \ll P^{3+\lambda / 3} R^{13 / 12-\tau / 4} Y^{1 / 6-\lambda / 3} .
$$

Since $Y=P^{11 / 79}$, we obtain $\mathcal{I}_{0} \ll P Q^{2} R^{2-\tau / 4} \Delta$, where

$$
\Delta=P^{37 / 102} R^{-11 / 12} Y^{7 / 51}=\left(P^{362 / 869} R^{-1}\right)^{11 / 12} .
$$

Thus, whenever $R \geq P^{\phi}$ and $P^{6 / 5} \leq X \leq P Y^{3}$, one finds from (5.1) that

$$
\sum_{N<n \leq 2 N}|\rho(n ; \mathfrak{K}(X))|^{2} \ll N^{2 \theta+8 / 9} R^{-\tau / 4} .
$$

The conclusion of the lemma now follows by summing over dyadic intervals covering the range $P^{6 / 5} \leq X \leq P Y^{3}$ for the parameter $X$, and then recalling the definition of $\phi$.

Lemma 5.3. Provided that $362 / 869 \leq \phi \leq 14 / 33-\tau / 6$, one has

$$
\sum_{N<n \leq 2 N}|\rho(n ; \mathfrak{U})|^{2} \ll N^{2 \theta+8 / 9} L^{-1 / 8}
$$


Proof. By Bessel's inequality, it follows from (4.1) that

$$
\sum_{N<n \leq 2 N}|\rho(n ; \mathfrak{U})|^{2} \leq \mathcal{J}_{0},
$$

where

$$
\mathcal{J}_{0}=\int_{\mathfrak{U}}|f(\alpha) \widetilde{K}(\alpha) g(\alpha) h(\alpha)|^{2} d \alpha .
$$

We define the function $f^{*}(\alpha)$ for $\alpha \in[0,1)$ by putting

$$
f^{*}(\alpha)=q^{-1} S(q, a) v(\alpha-a / q)
$$

when $\alpha \in \mathfrak{M}\left(q, a ; P^{6 / 5}\right) \subseteq \mathfrak{M}\left(P^{6 / 5}\right)$, and otherwise by taking $f^{*}(\alpha)=0$. Referring once again to (4.3), and noting that $\mathfrak{U} \subseteq \mathfrak{M}\left(P^{6 / 5}\right)$, we find that when $\alpha \in \mathfrak{U}$, one has

$$
f(\alpha)-f^{*}(\alpha) \ll P^{3 / 5+\varepsilon},
$$

whence

$$
\mathcal{J}_{0} \ll P^{6 / 5+\varepsilon} \mathcal{J}_{1}+\mathcal{J}_{2},
$$

where

$$
\mathcal{J}_{1}=\int_{0}^{1}|\widetilde{K}(\alpha) g(\alpha) h(\alpha)|^{2} d \alpha
$$

and

$$
\mathcal{J}_{2}=\int_{\mathfrak{U}}\left|f^{*}(\alpha) \widetilde{K}(\alpha) g(\alpha) h(\alpha)\right|^{2} d \alpha .
$$

But on considering the underlying diophantine equations, it follows from (2.5) that $\mathcal{J}_{1} \leq T_{3}$, and hence Lemma 2.4 implies that

$$
\mathcal{J}_{0} \ll P^{11 / 5+\varepsilon} Q R+\mathcal{J}_{2}
$$

Write

$$
v_{0}(\beta)=\int_{Q}^{2 Q} e\left(\beta \gamma^{3}\right) d \gamma,
$$

and define the function $g^{*}(\alpha)$ for $\alpha \in[0,1)$ by taking

$$
g^{*}(\alpha)=q^{-1} S(q, a) v_{0}(\alpha-a / q),
$$

when $\alpha \in \mathfrak{M}\left(q, a ; P^{6 / 5}\right) \subseteq \mathfrak{M}\left(P^{6 / 5}\right)$, and otherwise by setting $g^{*}(\alpha)=0$. Then it follows from [14, Theorem 4.1] that when $\alpha \in \mathfrak{M}\left(P^{6 / 5}\right)$, one has

$$
g(\alpha)-g^{*}(\alpha) \ll P^{3 / 5+\varepsilon} .
$$


On making use of this estimate within (5.10), we see that

$$
\mathcal{J}_{2} \ll P^{6 / 5+\varepsilon} \mathcal{J}_{3}+\mathcal{J}_{4},
$$

where

$$
\mathcal{J}_{3}=\int_{\mathfrak{U}}\left|f^{*}(\alpha) \widetilde{K}(\alpha) h(\alpha)\right|^{2} d \alpha
$$

and

$$
\mathcal{J}_{4}=\int_{\mathfrak{U}}\left|f^{*}(\alpha) g^{*}(\alpha) \widetilde{K}(\alpha) h(\alpha)\right|^{2} d \alpha .
$$

Next define $\Omega(\alpha)$ for $\alpha \in[0,1)$ by taking

$$
\Omega(\alpha)=\left(q+P^{3}|q \alpha-a|\right)^{-1},
$$

when $\alpha \in \mathfrak{M}\left(q, a ; P^{6 / 5}\right) \subseteq \mathfrak{M}\left(P^{6 / 5}\right)$, and otherwise by putting $\Omega(\alpha)=0$. Then an inspection of (5.9) leads from (4.8) and [14, Theorem 4.2] to the bound

$$
f^{*}(\alpha) \ll P \Omega(\alpha)^{1 / 3} \ll P^{6 / 5} \Omega(\alpha)^{1 / 2}
$$

On substituting this estimate into (5.13) and applying [2, Lemma 2], we deduce that

$$
\begin{aligned}
\mathcal{J}_{3} & \ll P^{12 / 5+\varepsilon} \int_{\mathfrak{M}\left(P^{6 / 5}\right)} \Omega(\alpha)|\widetilde{K}(\alpha) h(\alpha)|^{2} d \alpha \\
& \ll P^{\varepsilon-3 / 5}\left(P^{6 / 5} \int_{0}^{1}|\widetilde{K}(\alpha) h(\alpha)|^{2} d \alpha+P^{2} R^{2}\right) .
\end{aligned}
$$

A diminishing ranges argument akin to that establishing Lemma 2.1 therefore shows that whenever $P^{1 / 5} \leq R \leq P^{2 / 3}$, one has

$$
\mathcal{J}_{3} \ll P^{\varepsilon-3 / 5}\left(P^{11 / 5} R+P^{2} R^{2}\right) \ll P^{7 / 5+\varepsilon} R^{2} .
$$

Following the argument that led to the relation (2.14), one finds that when $\alpha \in \mathfrak{M}\left(q, a ; P^{6 / 5}\right) \subseteq \mathfrak{M}\left(P^{6 / 5}\right)$, one has

$$
g^{*}(\alpha) \ll Q q^{\varepsilon} \kappa(q) \quad \text { and } \quad f^{*}(\alpha) \ll P q^{1+\varepsilon} \kappa(q) \Omega(\alpha) .
$$

Consequently, under the same conditions on $\alpha$, one has

$$
f^{*}(\alpha) g^{*}(\alpha) \ll P Q q^{1+\varepsilon} \kappa(q)^{2} \Omega(\alpha) \ll P Q \Omega(\alpha)^{1 / 2} .
$$


In particular, whenever $\alpha \in \mathfrak{U}$, one has $f^{*}(\alpha) g^{*}(\alpha) \ll P Q L^{-1 / 2}$. An application of Hölder's inequality therefore leads from (5.14) to the estimate

$$
\mathcal{J}_{4} \ll h(0)^{2}\left(P Q L^{-1 / 2}\right)^{1 / 4} \mathcal{J}_{5}^{1 / 4} \mathcal{J}_{6}^{3 / 4}
$$

where

$$
\mathcal{J}_{5}=\int_{0}^{1}|\widetilde{K}(\alpha)|^{8} d \alpha \quad \text { and } \quad \mathcal{J}_{6}=\int_{\mathfrak{U}}\left|f^{*}(\alpha) g^{*}(\alpha)\right|^{7 / 3} d \alpha
$$

From [11, Theorem 2], one finds that $\mathcal{J}_{5} \ll P^{5}$. Meanwhile, a direct computation reveals that

$$
\begin{aligned}
\mathcal{J}_{6} & \ll(P Q)^{7 / 3}\left(\sum_{q=1}^{\infty} q^{1+\varepsilon} \kappa(q)^{14 / 3}\right) \int_{-\infty}^{\infty}\left(1+P^{3}|\beta|\right)^{-7 / 3} d \beta \\
& \ll(P Q)^{7 / 3} P^{-3} .
\end{aligned}
$$

Thus, it follows from (5.16) that

$$
\mathcal{J}_{4} \ll R^{2}\left(P Q L^{-1 / 2}\right)^{1 / 4}\left(P^{5}\right)^{1 / 4}\left((P Q)^{7 / 3} P^{-3}\right)^{3 / 4} \ll P Q^{2} R^{2} L^{-1 / 8}
$$

On combining (5.17) with (5.12) and (5.15) and then resubstituting into (5.11), we see that

$$
\mathcal{J}_{0} \ll P^{11 / 5+\varepsilon} Q R+P^{13 / 5+\varepsilon} R^{2}+P Q^{2} R^{2} L^{-1 / 8} \ll P Q^{2} R^{2} L^{-1 / 8},
$$

and the conclusion of the lemma now follows from (5.8).

The estimate (4.10) is obtained by combining the conclusions of Lemmas 5.1, 5.2, and 5.3. We have $\mathfrak{p} \subseteq \mathfrak{m} \cup \mathfrak{U} \cup \mathfrak{V}$, and so

$$
\begin{aligned}
\sum_{N<n \leq 2 N}|\rho(n ; \mathfrak{p})|^{2} & \ll \sum_{N<n \leq 2 N}\left(|\rho(n ; \mathfrak{m})|^{2}+|\rho(n ; \mathfrak{U})|^{2}+|\rho(n ; \mathfrak{V})|^{2}\right) \\
& \ll N^{2 \theta+8 / 9}\left(L^{-10}+L^{-1 / 8}\right) .
\end{aligned}
$$

In view of the concluding remarks of Section 4, the proof of Theorem 1.1 is now complete. 


\section{$\S 6$. The asymptotic formula for sums of four cubes}

Our objective in this section is the proof of Theorem 1.2. Experts will find the argument straightforward, though there are some technical irritations. We begin by adjusting some of our earlier notation in order to fit the circumstances at hand. Let $N$ be a large positive number, and write $P=(N / 4)^{1 / 3}$ and $L=(\log P)^{100}$. We consider a parameter $\theta$ with $0 \leq \theta<1 / 3$, and we take $R$ to be a number with $N^{\theta} \leq R \leq(2 N)^{\theta}$. Define $\sigma(n)=\sigma_{\theta}(n)$ to be the number of integral representations of $n$ in the form

$$
n=x_{1}^{3}+x_{2}^{3}+x_{3}^{3}+y^{3},
$$

with $x_{i} \in \mathbb{N}(i=1,2,3)$ and $1 \leq y \leq R$. Notice that when $N<n \leq 2 N$, then given any representation of $n$ in the shape (6.1), one has

$$
1 \leq x_{i} \leq(2 N)^{1 / 3} \quad(i=1,2,3) \quad \text { and } \quad \max _{1 \leq i \leq 3} x_{i}>(N / 4)^{1 / 3} .
$$

Thus,

$$
1 \leq x_{i} \leq 2 P \quad(i=1,2,3) \quad \text { and } \quad \max _{1 \leq i \leq 3} x_{i}>P .
$$

We define

$$
F(\alpha)=\sum_{1 \leq x \leq 2 P} e\left(\alpha x^{3}\right), \quad F_{0}(\alpha)=\sum_{1 \leq y \leq P} e\left(\alpha y^{3}\right),
$$

and

$$
G(\alpha)=\sum_{1 \leq z \leq R} e\left(\alpha z^{3}\right) .
$$

Then, given a measurable set $\mathfrak{B} \subseteq[0,1)$, we define

$$
\sigma(n ; \mathfrak{B})=\int_{\mathfrak{B}}\left(F(\alpha)^{3}-F_{0}(\alpha)^{3}\right) G(\alpha) e(-n \alpha) d \alpha .
$$

Notice that by orthogonality, one has $\sigma_{\theta}(n)=\sigma(n ;[0,1))$ whenever $N<$ $n \leq 2 N$. Next we define the set of $\operatorname{arcs} \mathfrak{P}$ as in Section 4 , and we take $\mathfrak{N}=\mathfrak{M}\left(P^{3 / 4}\right)$ and $\mathfrak{n}=[0,1) \backslash \mathfrak{N}$. Finally, we recall the familiar singular series $\mathfrak{S}(n)$ associated with sums of four cubes defined above in (4.7).

LEMMA 6.1. One has

$$
\sigma(n ; \mathfrak{P})=\Gamma\left(\frac{4}{3}\right)^{3} \mathfrak{S}(n) R+O\left(R L^{-1 / 16}\right)
$$

for all integers $n$ satisfying $N<n \leq 2 N$, with at most $O\left(N L^{-1 / 16}\right)$ possible exceptions. 
Proof. Write

$$
\begin{aligned}
& w_{0}(\beta)=\int_{0}^{P} e\left(\beta \gamma^{3}\right) d \gamma, \quad w_{1}(\beta)=\int_{0}^{2 P} e\left(\beta \gamma^{3}\right) d \gamma \\
& w_{2}(\beta)=\int_{0}^{R} e\left(\beta \gamma^{3}\right) d \gamma
\end{aligned}
$$

Also, define the functions $F^{*}(\alpha), F_{0}^{*}(\alpha)$, and $G^{*}(\alpha)$ for $\alpha \in[0,1)$ by putting

$$
F^{*}(\alpha)=q^{-1} S(q, a) w_{1}(\beta), \quad F_{0}^{*}(\alpha)=q^{-1} S(q, a) w_{0}(\beta),
$$

and

$$
G^{*}(\alpha)=q^{-1} S(q, a) w_{2}(\beta)
$$

when $\alpha \in \mathfrak{P}(q, a) \subseteq \mathfrak{P}$, and otherwise set each function to be zero. Then as a consequence of [14, Theorem 4.1], when $\alpha \in \mathfrak{P}$ one has

$$
F(\alpha)-F^{*}(\alpha) \ll L^{1 / 2+\varepsilon}, \quad F_{0}(\alpha)-F_{0}^{*}(\alpha) \ll L^{1 / 2+\varepsilon},
$$

and

$$
G(\alpha)-G^{*}(\alpha) \ll L^{1 / 2+\varepsilon}
$$

Now define $T(q, a)$ as in (4.4), and put

$$
W(\beta)=\left(w_{1}(\beta)^{3}-w_{0}(\beta)^{3}\right) w_{2}(\beta) .
$$

Then we see that for $\alpha \in \mathfrak{P}(q, a) \subseteq \mathfrak{P}$, one has

$$
\left(F(\alpha)^{3}-F_{0}(\alpha)^{3}\right) G(\alpha)-T(q, a) W(\alpha-a / q) \ll P^{3} L^{1 / 2+\varepsilon} .
$$

Since the measure of $\mathfrak{P}$ is $O\left(L^{3} N^{-1}\right)$, we deduce from $(6.2)$ that

$$
\sigma(n ; \mathfrak{P})-\mathfrak{S}(n ; L) \mathcal{J}_{0}(n ; L) \ll L^{7 / 2+\varepsilon},
$$

where $\mathfrak{S}(n ; L)$ is defined as in $(4.6)$, and

$$
\mathcal{J}_{0}(n ; L)=\int_{-L / N}^{L / N} W(\beta) e(-\beta n) d \beta .
$$

The series $\mathfrak{S}(n ; L)$ has already been considered during the course of the proof of Lemma 4.1. In particular, it follows that for all integers $n$ with $N<n \leq 2 N$, one has

$$
|\mathfrak{S}(n ; L)-\mathfrak{S}(n)| \leq L^{-1 / 16},
$$


with at most $O\left(N L^{-1 / 16}\right)$ possible exceptions. In addition, for all natural numbers $n$, one has $1 \ll \mathfrak{S}(n) \ll(\log \log n)^{4}$. Note that the former conclusion is robust to the adjustment in our definition of $L$. Next, recall definition (4.2). Then we see that

$$
\begin{aligned}
W(\beta) & =\left(\left(v(\beta)+w_{0}(\beta)\right)^{3}-w_{0}(\beta)^{3}\right) w_{2}(\beta) \\
& =\left(v(\beta)^{3}+3 v(\beta)^{2} w_{0}(\beta)+3 v(\beta) w_{0}(\beta)^{2}\right) w_{2}(\beta) .
\end{aligned}
$$

Then by (4.8) and the bound $w_{0}(\beta) \ll P\left(1+P^{3}|\beta|\right)^{-1 / 3}$ that follows from [14, Theorem 7.3], we see that

$$
W(\beta) \ll P^{3} R\left(1+P^{3}|\beta|\right)^{-5 / 3} .
$$

Consequently, one has

$$
\mathcal{J}_{0}(n ; L)-\int_{-\infty}^{\infty} W(\beta) e(-\beta n) d \beta \ll P^{3} R N^{-1} L^{-2 / 3} .
$$

The last integral may be evaluated in a standard manner (see [7, pages 21, 22]). Thus we see that when $N<n \leq 2 N$, one has

$$
\mathcal{J}_{0}(n ; L)=\Gamma\left(\frac{4}{3}\right)^{3} R+O\left(R L^{-2 / 3}\right)
$$

(compare the discussion in [18, Section 4]). On substituting these estimates into (6.3), we find that

$$
\sigma(n ; \mathfrak{P})=\Gamma\left(\frac{4}{3}\right)^{3} \mathfrak{S}(n) R+O\left(R L^{-1 / 16}\right)
$$

for all integers $n$ satisfying $N<n \leq 2 N$, with at most $O\left(N L^{-1 / 16}\right)$ possible exceptions. The conclusion of the lemma follows.

Lemma 6.2. Provided that $1 / 4<\theta<1 / 3$, one has

$$
\sum_{N<n \leq 2 N}|\sigma(n ; \mathfrak{p})|^{2} \ll N R^{2} L^{\varepsilon-1 / 3}
$$

Proof. An application of Bessel's inequality leads from (6.2) to the upper bound

$$
\begin{aligned}
\sum_{N<n \leq 2 N}|\sigma(n ; \mathfrak{p})|^{2} & \ll \sum_{N<n \leq 2 N}|\sigma(n ; \mathfrak{n})|^{2}+\sum_{N<n \leq 2 N}|\sigma(n ; \mathfrak{N} \backslash \mathfrak{P})|^{2} \\
& \leq \Xi(\mathfrak{n})+\Xi(\mathfrak{N} \backslash \mathfrak{P}),
\end{aligned}
$$


where for a measurable set $\mathfrak{B}$, we write

$$
\Xi(\mathfrak{B})=\int_{\mathfrak{B}}\left|\left(F(\alpha)^{3}-F_{0}(\alpha)^{3}\right) G(\alpha)\right|^{2} d \alpha .
$$

It follows from (2.1) that

$$
\int_{\mathfrak{n}}\left|\left(F(\alpha)^{3}-F_{0}(\alpha)^{3}\right) G(\alpha)\right|^{2} d \alpha \ll \int_{\mathfrak{n}}|f(\alpha) G(\alpha)|^{2}\left(|F(\alpha)|^{4}+\left|F_{0}(\alpha)\right|^{4}\right) d \alpha,
$$

and so an application of Hölder's inequality reveals that

$$
\Xi(\mathfrak{n}) \ll\left(\sup _{\alpha \in \mathfrak{n}}|f(\alpha)|\right)\left(\mathcal{K}_{1}+\mathcal{K}_{2}\right)^{1 / 2} \mathcal{K}_{3}^{1 / 2}
$$

where

$$
\mathcal{K}_{1}=\int_{0}^{1}|F(\alpha)|^{8} d \alpha, \quad \mathcal{K}_{2}=\int_{0}^{1}\left|F_{0}(\alpha)\right|^{8} d \alpha
$$

and

$$
\mathcal{K}_{3}=\int_{0}^{1}\left|f(\alpha)^{2} G(\alpha)^{4}\right| d \alpha .
$$

But a modified version of Weyl's inequality (see, e.g., [11, Lemma 1]) confirms that

$$
\sup _{\alpha \in \mathfrak{n}}|f(\alpha)| \ll P^{3 / 4+\varepsilon} \text {. }
$$

Thus, on recalling Lemma 2.3 and applying Hua's lemma (see, e.g., [14, Lemma 2.5]), one finds that

$$
\begin{aligned}
\Xi(\mathfrak{n}) & \ll P^{3 / 4+\varepsilon}\left(P^{5}\right)^{1 / 2}\left(P R^{2}+P^{-1} R^{9 / 2}\right)^{1 / 2} \\
& \ll P^{3+\varepsilon} R^{2}\left(P^{3 / 4} R^{-1}+(R / P)^{1 / 4}\right) .
\end{aligned}
$$

It remains to estimate the contribution arising from the set of arcs $\mathfrak{N} \backslash \mathfrak{P}$. Here one may appeal to standard major arc technology (see [12, Lemma 5.1]) to show that

$$
\begin{aligned}
\Xi(\mathfrak{N} \backslash \mathfrak{P}) & \ll \sum_{1 \leq z_{1}, z_{2} \leq R} \int_{\mathfrak{N} \backslash \mathfrak{P}}|F(\alpha)|^{6} e\left(\alpha\left(z_{1}^{3}-z_{2}^{3}\right)\right) d \alpha \\
& +\sum_{1 \leq z_{1}, z_{2} \leq R} \int_{\mathfrak{N} \backslash \mathfrak{P}}\left|F_{0}(\alpha)\right|^{6} e\left(\alpha\left(z_{1}^{3}-z_{2}^{3}\right)\right) d \alpha \\
& \ll P^{3} R^{2} L^{\varepsilon-1 / 3} .
\end{aligned}
$$


On substituting (6.5) and (6.6) into (6.4), we therefore deduce that

$$
\sum_{N<n \leq 2 N}|\sigma(n ; \mathfrak{p})|^{2} \ll P^{3} R^{2} L^{\varepsilon-1 / 3}+P^{3+\varepsilon} R^{2}\left(P^{3 / 4} R^{-1}+(R / P)^{1 / 4}\right) .
$$

Consequently, when $1 / 4<\theta<1 / 3$, the conclusion of the lemma follows on recalling that $R=P^{3 \theta}$.

We finish this section with the proof of Theorem 1.2. Observe first that when $\theta=1 / 3$, the desired conclusion is an immediate consequence of [11, Theorem 3], provided that one makes use of the refinement to be found in the main theorem of Boklan [1]. We assume henceforth, therefore, that $1 / 4<\theta<1 / 3$. When $N^{\theta} \leq R \leq(2 N)^{\theta}$, with $\theta$ in the latter range, it follows from Lemma 6.2 that

$$
\sum_{N<n \leq N+N /(\log N)^{2}}|\sigma(n ; \mathfrak{p})|^{2} \ll P^{3} R^{2}(\log N)^{-6} .
$$

It follows, in particular, that $|\sigma(n ; \mathfrak{p})| \ll R(\log N)^{-1}$ for all integers $n$ with $N<n \leq N+N(\log N)^{-2}$, with at most $O\left(N(\log N)^{-4}\right)$ exceptions. In combination with the conclusion of Lemma 6.1 , this shows that

$$
\sigma_{\theta}(n)=\sigma(n ;[0,1))=\Gamma\left(\frac{4}{3}\right)^{3} \mathfrak{S}(n) R+O\left(R(\log N)^{-1}\right)
$$

for all $n$ with $N<n \leq N+N(\log N)^{-2}$, with at most $O\left(N(\log N)^{-4}\right)$ exceptions. Given an interval of the latter type for $n$, one has

$$
n^{\theta}=N^{\theta}+O\left(N^{\theta}(\log N)^{-2}\right) .
$$

There is therefore a positive constant $A$ with the property that

$$
N^{\theta}<n^{\theta} \leq N^{\theta}+A N^{\theta}(\log N)^{-2} .
$$

Taking $R$ to be first $N^{\theta}$, and then $N^{\theta}+A N^{\theta}(\log N)^{-2}$, we see that

$$
r_{\theta}(n)=\Gamma\left(\frac{4}{3}\right)^{3} \mathfrak{S}(n) N^{\theta}+O\left(N^{\theta}(\log N)^{-1}\right),
$$

whence

$$
r_{\theta}(n)=\Gamma\left(\frac{4}{3}\right)^{3} \mathfrak{S}(n) n^{\theta}+O\left(n^{\theta}(\log n)^{-1}\right),
$$

for all $n$ with $N<n \leq N+N(\log N)^{-2}$, with at most $O\left(N(\log N)^{-4}\right)$ exceptions. On summing over the $O\left((\log N)^{3}\right)$ intervals of this type that cover $[1,2 N]$, we find that the total number of exceptions encountered is $O\left(N(\log N)^{-1}\right)$, whence the conclusion of Theorem 1.2 follows also when $1 / 4<\theta<1 / 3$. 


\section{$\S 7$. Sums of six cubes and a minicube}

Thus far, our conclusions have been of almost-all type. We now briefly discuss the proof of the following theorem.

TheOREM 7.1. All large natural numbers $n$ are the sum of seven cubes of positive integers, one of which is no larger than $n^{43 / 168}$.

We require a simple lemma.

Lemma 7.2. Let $\mathcal{N}_{\theta}(X)$ denote the number of integers $n$ with $1 \leq n \leq X$ that are the sum of three cubes of natural numbers, one of which is at most $n^{\theta}$. Then whenever $0 \leq \theta \leq 5 / 18$, one has

$$
\mathcal{N}_{\theta}(X) \gg X^{5 / 8+\theta+\tau / 9}
$$

Proof. Write $P=(X / 20)^{1 / 3}$ and $R=(X / 20)^{\theta}$. Also, let $\nu(n)$ denote the number of solutions of the equation

$$
n=x_{1}^{3}+x_{2}^{3}+y^{3},
$$

with $x_{i} \in \mathcal{B}(P, P)$ and $1 \leq y \leq R$. Then by Cauchy's inequality, one has

$$
\mathcal{N}_{\theta}(X) \geq \sum_{\substack{X / 10 \leq n \leq X \\ \nu(n) \geq 1}} 1 \geq\left(\sum_{X / 10 \leq n \leq X} \nu(n)\right)^{2}\left(\sum_{1 \leq n \leq X} \nu(n)^{2}\right)^{-1} .
$$

Observe that

$$
\sum_{X / 10 \leq n \leq X} \nu(n) \gg P^{2} R
$$

Also, in view of the underlying diophantine equation, one has

$$
\sum_{1 \leq n \leq X} \nu(n)^{2} \leq \int_{0}^{1}\left|\mathcal{F}(\alpha)^{4} G(\alpha)^{2}\right| d \alpha,
$$

where we have written

$$
\mathcal{F}(\alpha)=\sum_{x \in \mathcal{B}(P, P)} e\left(\alpha x^{3}\right) .
$$

By Schwarz's inequality, we obtain

$$
\int_{0}^{1}\left|\mathcal{F}(\alpha)^{4} G(\alpha)^{2}\right| d \alpha \leq\left(\int_{0}^{1}|\mathcal{F}(\alpha)|^{6} d \alpha\right)^{1 / 2}\left(\int_{0}^{1}\left|\mathcal{F}(\alpha)^{2} G(\alpha)^{4}\right| d \alpha\right)^{1 / 2} .
$$


The first integral may be estimated via (2.2), and the second by means of Lemma 2.3. Thus we deduce that when $\theta \leq 5 / 18$, one has

$$
\sum_{1 \leq n \leq X} \nu(n)^{2} \ll\left(P^{13 / 4-\tau}\right)^{1 / 2}\left(P^{1+\varepsilon} R^{2}\right)^{1 / 2} .
$$

Finally, on substituting (7.2) and (7.3) into (7.1), we conclude that

$$
\mathcal{N}_{\theta}(X) \gg\left(P^{2} R\right)^{2}\left(P^{17 / 8-\tau / 3} R\right)^{-1} \gg P^{15 / 8+\tau / 3} R
$$

thereby completing the proof of the lemma.

Next, let $X$ be a sufficiently large positive number, and consider an integer $n$ with $X \leq n \leq 2 X$. By Lemma 7.2 , at least $X^{5 / 8+\theta+\tau / 9}$ of the integers $m$ with $1 \leq m \leq X$ are the sum of three cubes of natural numbers, one of which is at most $m^{\theta} \leq n^{\theta}$. But all the integers $n-m$, with $1 \leq m \leq X$, are the sum of four cubes of natural numbers, with at most $O\left(X^{37 / 42}\right)$ exceptions (see [17, Theorem 1.3]). Thus, provided that $X^{5 / 8+\theta+\tau / 9}>X^{37 / 42}$, then for at least one value of $m$ with $1 \leq m \leq X$, one finds that $m$ is the sum of three cubes of natural numbers, one at most $n^{\theta}$, and $n-m$ is the sum of four positive integral cubes, whence $n$ is the sum of six positive integral cubes and a minicube at most $n^{\theta}$. The conclusion of Theorem 7.1 follows on observing that the above condition is satisfied whenever $\theta \geq 43 / 168$.

Acknowledgments. This article was conceived at Kinki University in Osaka and gestated at the Hausdorff Research Institute in Bonn. The authors are grateful to both institutions for their generous hospitality. The authors are also grateful to the referee for useful comments.

\section{REFERENCES}

[1] K. D. Boklan, A reduction technique in Waring's problem, I, Acta Arith. 65 (1993), $147-161$.

[2] J. Brüdern, A problem in additive number theory, Math. Proc. Cambridge Philos. Soc. 103 (1988), 27-33.

[3] - Sums of four cubes, Monatsh. Math. 107 (1989), 179-188.

[4] - On Waring's problem for cubes, Math. Proc. Cambridge Philos. Soc. 109 (1991), 229-256.

[5] J. Brüdern and T. D. Wooley, On Waring's problem: three cubes and a sixth power, Nagoya Math. J. 163 (2001), 13-53.

[6] H. Davenport, On Waring's problem for cubes, Acta Math. 71 (1939), 123-143.

[7] — Analytic Methods for Diophantine Equations and Diophantine Inequalities, 2nd ed., Cambridge University Press, Cambridge, 2005. 
[8] R. A. Hunt, "On the convergence of Fourier series" in Proceedings of the Conference on Orthogonal Expansions and Their Continuous Analogues (Edwardsville, Ill., 1967), Southern Illinois University Press, Carbondale, Illinois, 1968, 235-255.

[9] K. Kawada, On the sum of four cubes, Mathematika 43 (1996), 323-348.

[10] R. C. Vaughan, Sums of three cubes, Bull. Lond. Math. Soc. 17 (1985), 17-20.

[11] - On Waring's problem for cubes, J. Reine Angew. Math. 365 (1986), 122-170.

[12] — A new iterative method in Waring's problem, Acta Math. 162 (1989), 1-71.

[13] - On Waring's problem for cubes, II, J. Lond. Math. Soc. (2) 39 (1989), 205218.

[14] — The Hardy-Littlewood Method, 2nd ed., Cambridge University Press, Cambridge, 1997.

[15] T. D. Wooley, On simultaneous additive equations, II, J. Reine Angew. Math. 419 (1991), 141-198.

[16] - Breaking classical convexity in Waring's problem: sums of cubes and quasidiagonal behaviour, Invent. Math. 122 (1995), 421-451.

[17] - Sums of three cubes, Mathematika 47 (2000), 53-61.

[18] - A light-weight version of Waring's problem, J. Austral. Math. Soc. Ser. A 76 (2004), 303-316.

Jörg Brüdern

Mathematisches Institut

D-37073 Göttingen

Germany

bruedern@uni-math.gwdg.de

Trevor D. Wooley

School of Mathematics

University of Bristol

Bristol BS8 $1 T W$

United Kingdom

matdw@bristol . ac .uk 